\title{
Free-Standing 2D Janus Gold nanoparticles Monolayer Film with Tunable Bifacial Morphologies via the Asymmetric Growth at Air-liquid Interface
}

Qian Cheng, a, biping Song, a, b Han Lin, ${ }^{\text {a } Y a n p i n g ~ Y a n g, ~}{ }^{\text {a }}$ Youju Huang, ${ }^{*}$ a, b, c Fengmei Su, ${ }^{d}$ and Tao Chen ${ }^{* a, b}$

a. Key Laboratory of Marine Materials and Related Technologies, Zhejiang Key Laboratory of Marine Materials and Protective Technologies, Ningbo Institute of Materials Technology and Engineering, Chinese Academy of Sciences, Ningbo 315201, China

b. University of Chinese Academy of Sciences, 19A Yuquan Road, Beijing 100049, China

c. College of Materials, Chemistry and Chemical Engineering, Hangzhou Normal University, Hangzhou, Zhejiang 311121, China

d. National Engineering Research Centre for Advanced Polymer Processing Technology, Key Laboratory of Materials Processing and Mold (Zhengzhou University), Ministry of Education, Zhengzhou University, Zhengzhou 450002, P.R. China.

${ }^{*}$ Corresponding Authors: E-mail: yjhuang@nimte.ac.cn; and tao.chen@,nimte.ac.cn. 
(A)

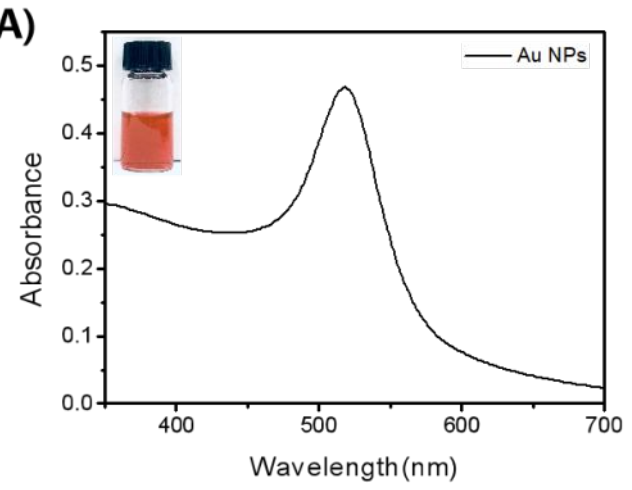

(D)

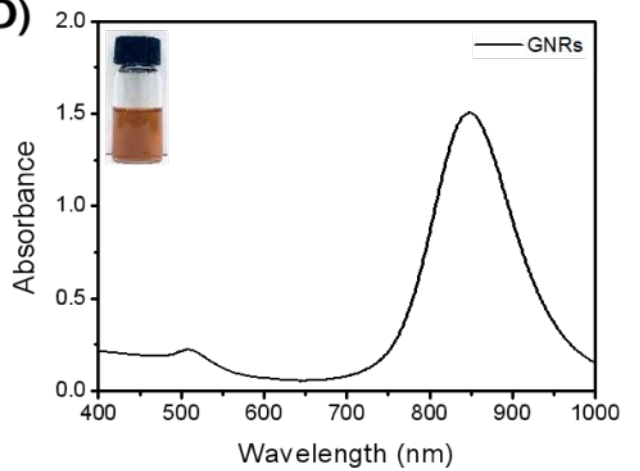

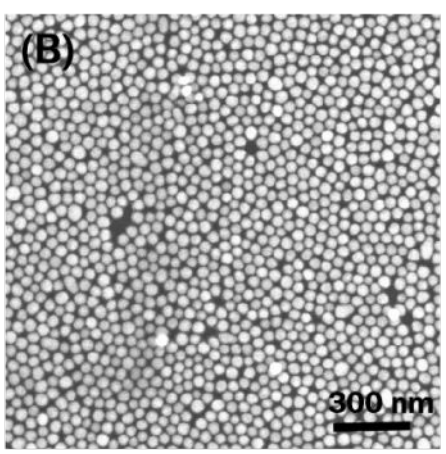
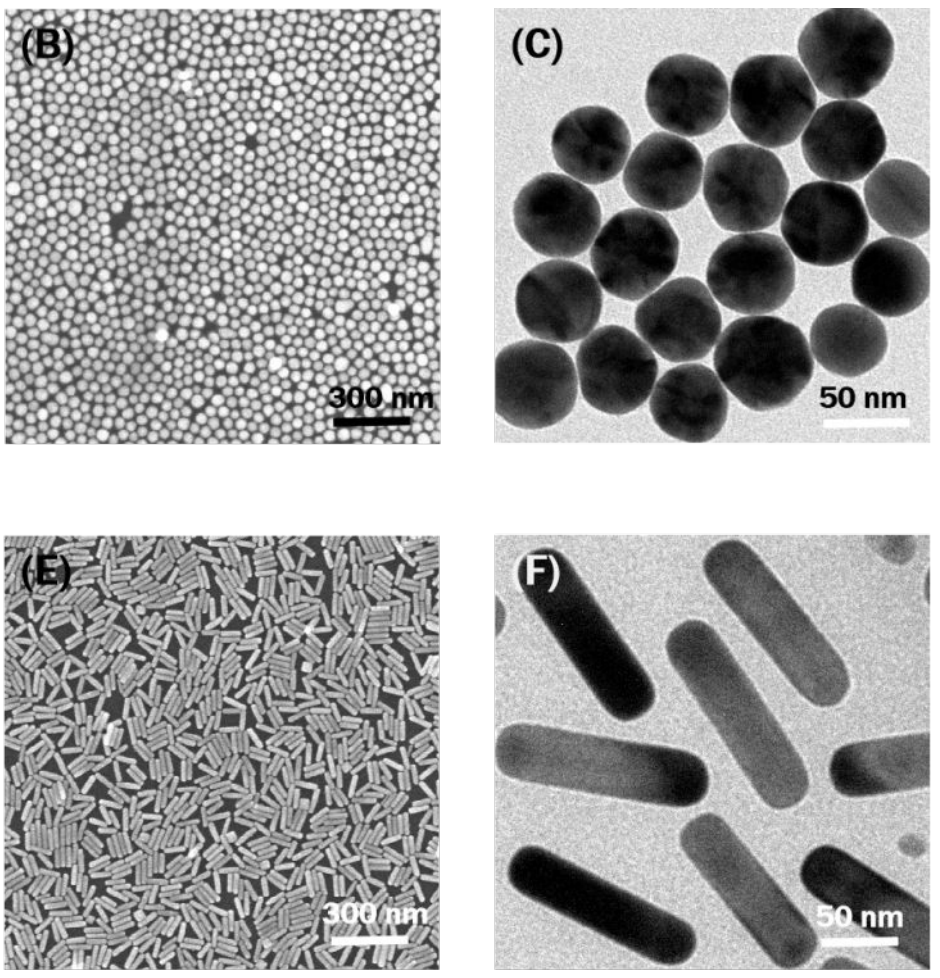

Figure S1. Representative UV-vis-NIR absorption spectra (A)(D), SEM images (B)(E) and TEM images (C)(F) of gold nanoparticles (Au NPs) with $50 \mathrm{~nm}$ in size and gold nanorods (GNRs) with aspect ratio of 4 to 1 used in experiments.
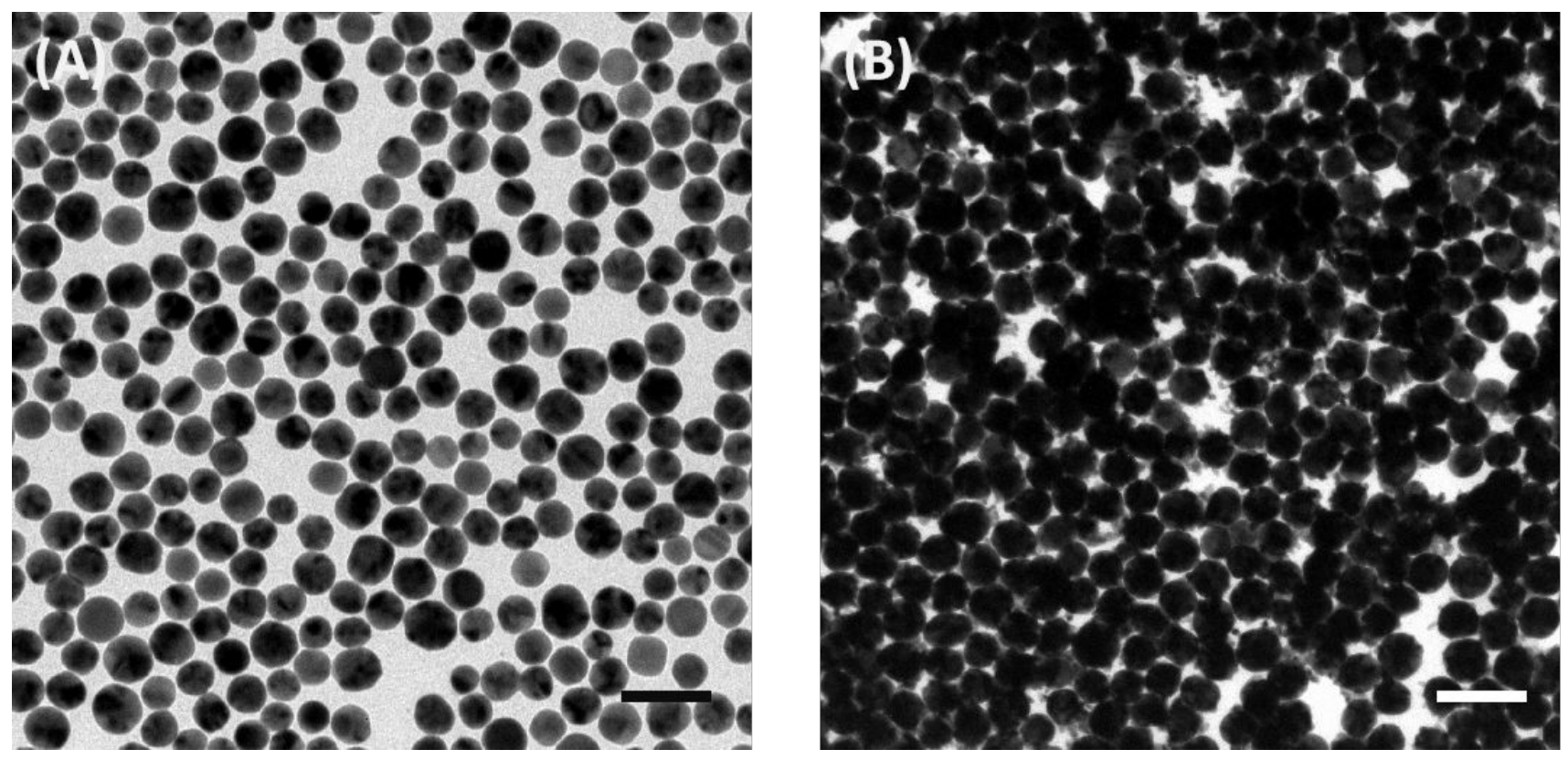

Figure S2. TEM images of the original gold nanoparticles before asymmetric growth (A) and the top surface of Janus film after growth in $350 \mu \mathrm{M}$ 4-MBA in the reaction solution (B). scale bar is $100 \mathrm{~nm}$. 

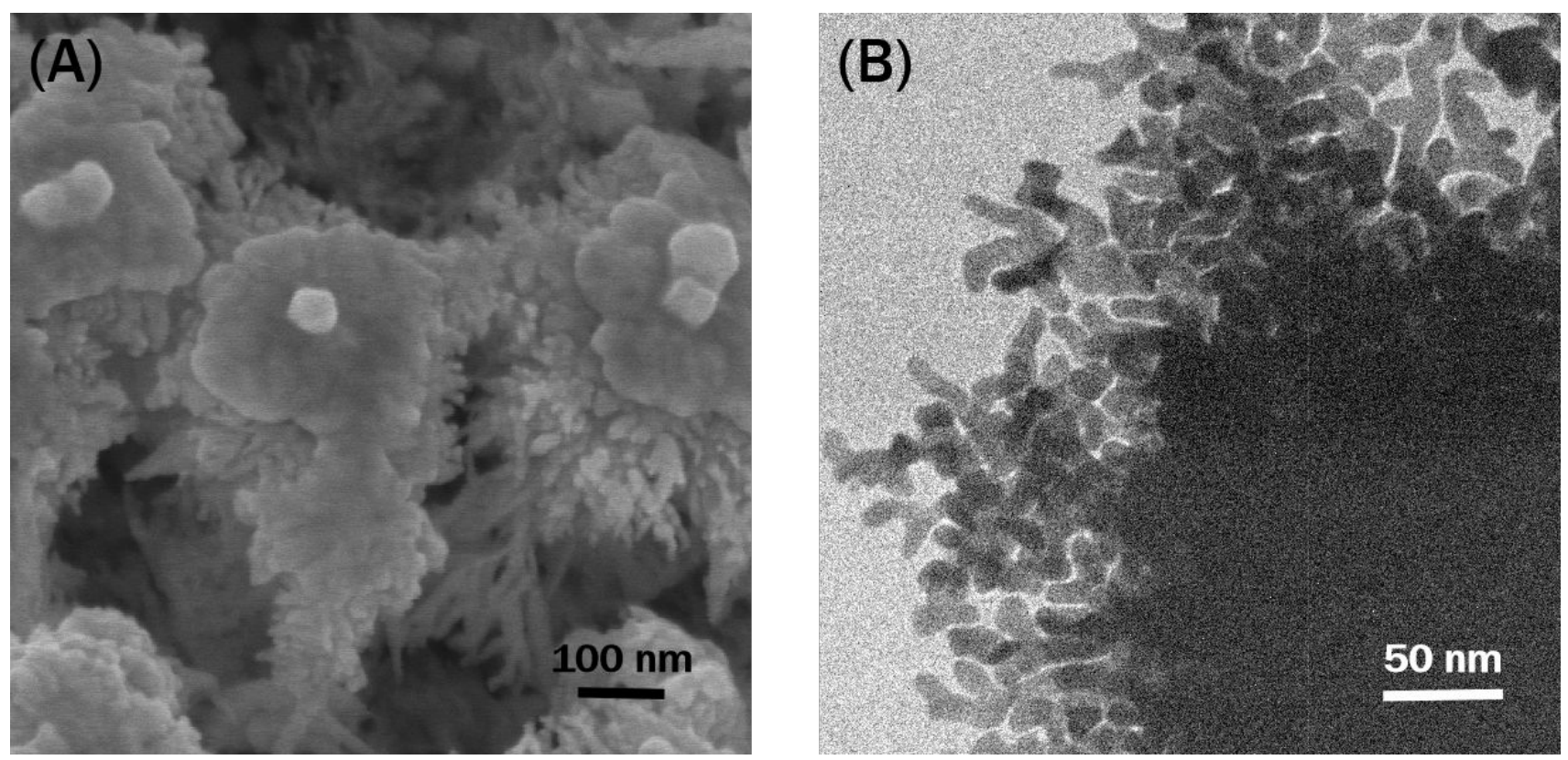

Figure S3. SEM (A) and TEM (B) images of the bottom surface of individual Janus nanoparticles in $25 \mu \mathrm{M}$ 4-MBA in the reaction solution.

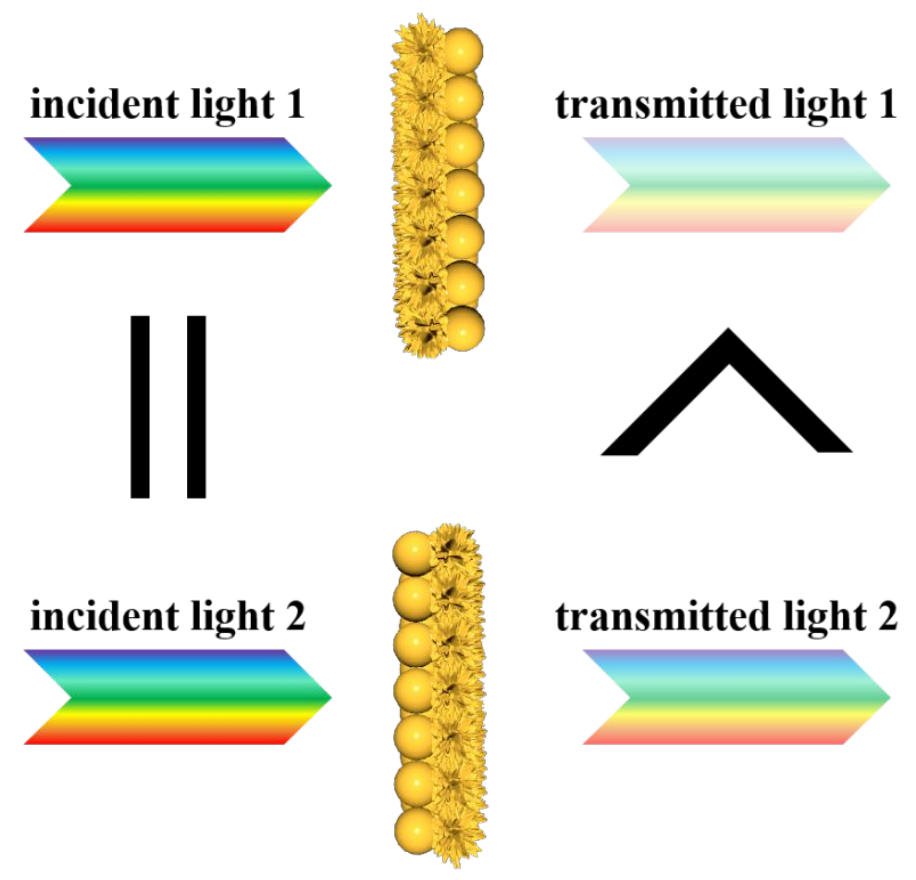

Figure S4. Schematic illustration of the method of measuring optical properties of the Janus monolayer film of gold nanoparticles. 